\title{
Resistencia al clima tropical de aceros galvanizados con y sin recubrimiento
}

\section{Tropical Weather Resistant Galvanized Steel Coated and Uncoated}

\author{
Suárez-Corrales Xenia Isbel \\ Laboratorio de Ensayos de Tropicalización \\ Centro de Servicios Tecnológicos \\ La Habana, Cuba \\ Correo:xenia@labet.co.cu \\ Villar-López René Valentino \\ Laboratorio de Ensayos de Tropicalización \\ Centro de Servicios Tecnológicos \\ La Habana, Cuba \\ Correo:villar@labet.co.cu
}

\author{
Corvo-Pérez Francisco Eduardo \\ Centro de Investigación en Corrosión \\ Universidad Autónoma de Campeche \\ Correo:corvo.francisco@gmail.com \\ Marrero Rigoberto \\ Facultad de Química \\ Instituto Superior Politéenico José A. Echeverría \\ La Habana, Cuba \\ Correo: rigo@quimica.cujae.edu.cu
}

Información del artículo: recibido: enero de 2012, reevaluado: julio de 2012, aceptado: marzo de 2013

\section{Resumen}

En el presente trabajo se evalúa el comportamiento a la corrosión de diferentes productos de acero galvanizado con y sin recubrimiento para las condiciones de un clima tropical húmedo, mediante ensayos climáticos acelerados efectuados en cámaras climáticas y ensayos naturales en la estación marino-costera de Cojímar, en donde quedaron en evidencia los problemas que suelen presentar los aceros galvanizados al ser expuestos con y sin recubrimiento, en un ambiente tropical húmedo, así como la importancia de la adherencia para su eficaz comportamiento, bien sea con fines decorativos o para impartir una protección adicional. Los resultados reflejan que 95\% del acero galvanizado estudiado en el presente trabajo presenta una buena resistencia a la corrosión y $71 \%$ de los aceros galvanizados con recubrimientos aplicados y de ellos, $100 \%$ de los aceros galvanizados pintados no cumplen las expectativas para las que fueron concebidos desde el punto de vista mecánico y corrosivo. Por tanto, se evidencia que es vital para la eficiencia y rentabilidad de la industria metal-mecánica expuesta al clima tropical húmedo, lograr elementos de acero galvanizado pintado con las debidas preparaciones para la superficie y la aplicación del recubrimiento, constituyendo estos puntos la clave que incide en el logro de las propiedades que precisa este producto para garantizar su efectividad y un mejor comportamiento a la corrosión que el acero galvanizado sin pintar, prolongando así su durabilidad.

\section{Descriptores:}

- corrosión

- acero galvanizado

- recubrimientos

- clima tropical 


\begin{abstract}
The corrosion behavior of coated and uncoated galvanized steel products is determined by exposing them to accelerated and natural climatic test. Accelerated tests were carried out in climatic chambers and natural atmospheric test in a marinecoastal station (Cojimar). The influence of tropical humid climate on these products is determined. Adhesion is an important property for a good performance, from the decorative point of view or as an additional protection. The evaluation indicates that 95\% of the galvanized steel products show a good corrosion resistance; although $71 \%$ of coated galvanized products and $100 \%$ of painted galvanized products do not cover the requirements with respect to the mechanical and corrosion protection aspects. For a good efficiency and economic performance of metal mechanical industry, a correct surface treatment and coating application is required. A key point is to increase the quality by improving a better corrosion protective performance respecting uncoated galvanized steel.
\end{abstract}

\section{Introducción}

Tradicionalmente el recubrimiento galvanizado se ha venido considerando como un sistema de protección del acero eficaz y duradero que no necesita tratamiento adicional alguno. La galvanización por inmersión en caliente es el recubrimiento industrial de protección comúnmente utilizado en la fabricación de piezas y componentes expuestos en ambientes, incluso de alta agresividad atmosférica, debido a su alto índice de fiabilidad. Al ser un recubrimiento obtenido por inmersión en zinc fundido, cubre la totalidad de la superficie de las piezas exteriores e interiores de las partes huecas y otras áreas superficiales que no son accesibles por otros métodos de protección.

Es bien conocido que el recubrimiento galvanizado le otorga al acero una excelente protección, aportándole una serie de propiedades entre las que se encuentra su gran resistencia a la abrasión y a la corrosión. La duración de la protección que proporcionan los recubrimientos galvanizados frente a la corrosión atmosférica es extremadamente alta y depende de las condiciones climatológicas del lugar y de la presencia en la atmósfera de contaminantes agresivos, como son los óxidos de azufre y los cloruros, éstos últimos típicos de las zonas costeras (Leygraf y Graedel, 2000).

El zinc presenta una buena resistencia a la corrosión, debido a que tiene la propiedad de formar una película compacta y de buena adherencia sobre el hierro, constituida por sus productos de corrosión (Zhu et al., 2000). Al ser el zinc un metal más activo que el hierro, da lugar a la formación de un par galvánico con el acero, disminuyendo así su velocidad de deterioro (Granesse y Rosales, 1986), confirmándose que el depósito superficial de zinc incrementa la vida útil del acero. No obstante, el poder protector del zinc depende de nu- merosos factores, entre los que se destacan el espesor y la porosidad de la capa de zinc, la adherencia al hierro, la naturaleza de los productos de corrosión formados, así como del tiempo de exposición al medio, los factores climáticos y contaminantes atmosféricos (Townsend y Gorman, 1999; Costa y Vilarrasca, 1993). Para acero y zinc, humedades relativas de 70 y $80 \%$, respectivamente, marcan el nivel mínimo necesario para la corrosión, y las precipitaciones pluviales desplazan el equilibrio de las reacciones al lavar los productos de corrosión formados, produciéndose el electrolito necesario para las reacciones electro-químicas (Vázquez y Damborenea, 1992).

La estabilidad del zinc en un medio agresivo depende de las propiedades protectoras de la película que desarrolla, cuya composición química y estructura determinan su comportamiento como barrera que controla el tipo de ataque y la velocidad de corrosión. El producto inicial de la corrosión de zinc en la atmósfera es la formación de óxido de zinc caracterizado por ser una película muy dura, que al reaccionar con la humedad del aire, forma el hidróxido de zinc $\left(\mathrm{Zn}(\mathrm{OH})_{2}\right)$ como segundo producto de corrosión. Posteriormente, este reacciona con $\mathrm{CO}_{2}$ de la atmósfera para formar un carbonato básico de zinc, generalmente de la forma $2 \mathrm{ZnCO}_{3}{ }^{*} 3 \mathrm{Zn}(\mathrm{OH})_{2}$. Esta película de carbonato básico de zinc es altamente adherente, relativamente insoluble y, lo más importante, es responsable de la protección contra la corrosión del zinc en variadas condiciones atmosféricas (Ahmed et al., 2000).

Actualmente se recurre con más frecuencia al pintado del acero galvanizado, sobretodo después de que los estudios de corrosión del acero galvanizado y pintado han demostrado que la combinación de recubrimiento galvanizado más pintura proporciona protección por un periodo que es de 1.8 a 2.2 veces superior a la suma 
de la duración de cada sistema de protección por separado. Este efecto tan favorable se explica porque en toda capa de pintura se forman, antes o después, fisuras o pequeñas zonas desnudas que constituyen los puntos de iniciación de su destrucción, ya que la oxidación del acero que se inicia en ellos progresa por debajo de la pintura y da lugar a su levantamiento. Cuando la pintura no es completamente impermeable no es necesario siquiera la aparición inicial de estos puntos desnudos. Sobre el acero galvanizado los deterioros iniciales de la pintura se producen igualmente, pero no es posible que progrese la oxidación por debajo de la película de pintura porque en el fondo de las fisuras o poros de la misma se forman depósitos con los productos de corrosión de la capa de zinc que son insolubles, compactos y adherentes, los cuales taponan estos defectos e impiden la subsiguiente penetración de la humedad. Por este motivo, muchos técnicos en protección del acero, proyectistas, etcétera consideran que la galvanización constituye, desde el punto de vista de la seguridad frente a la corrosión, una buena preparación para la superficie del acero antes de pintarlo.

El clima tropical húmedo se reconoce como un entorno agresivo para metales, debido a los valores anuales muy altos de tiempo de humedad para las estructuras metálicas, lo que proporciona las condiciones para mayor rapidez de corrosión, incluso en presencia de bajos niveles de contaminantes, en comparación con diferentes climas templados (Veleva et al., 2009). El galvanizado expuesto a la intemperie en ambientes corrosivos se deteriora con el tiempo. Por ende, la pintura aplicada, dependiendo el ambiente, evita su deterioro, pues el galvanizado sólo resulta expuesto en el momento que la pintura falla y es en ese momento que desarrolla su acción anticorrosiva; además de la posibilidad de poder lograrse el aspecto estético deseado, sin tener que quedar limitado al gris metálico propio del galvanizado. Aunque el galvanizado se utiliza extensivamente en la fabricación de una gran variedad de productos que requieren protección contra la corrosión, sus usos principales están en el acero estructural utilizado en obras públicas y viales, torres de transmisión y comunicaciones y estructuras en áreas químicas, construcción, marina, agrícola, minera, etcétera (Morcillo et al., 2002).

Como se sabe, la protección de barrera como son las pinturas tienen la desventaja que si la capa de pintura se rompe de alguna forma se oxidará el acero en esa área y la pintura permitirá que la oxidación avance por debajo de la ruptura. En el caso del galvanizado esto no ocurre, ya que si la capa de galvanizado se daña, raya o presenta discontinuidades, el zinc adyacente al acero formará una sal insoluble de zinc sobre el acero expuesto. Esto resana la ruptura y continúa protegiendo la superficie contra cualquier corrosión. No obstante, es importante considerar que cuando se produzcan discontinuidades en la película de pintura, por envejecimiento o por daño mecánico, el recubrimiento galvanizado ejercerá su acción protectora y se irá consumiendo gradualmente a una velocidad que estará determinada por las condiciones de exposición (Vera, 2005).

Por todo lo expuesto, el objetivo del presente trabajo es evaluar el comportamiento de diferentes muestras de acero galvanizado y acero galvanizado pintado, que por sus condiciones de explotación deben ser sometidos a las características del clima tropical húmedo, considerando la salinidad como contaminante fundamental, en búsqueda de evidenciar la tendencia y características técnicas que debe regir la selección de estos materiales.

\section{Materiales y métodos experimentales}

El presente trabajo se efectúa sobre la base de una población conformada por 29 productos empleados en sistemas constructivos, los cuales fueron seleccionados aleatoriamente de muestras entregadas por los productores o comercializadores antes de su explotación, en las cuales estarán expuestas a los efectos de la temperatura, la humedad y la salinidad. Tienen un material base común de acero al carbono de baja aleación laminado en frío o caliente, apto para uso estructural, recubierto en ambas caras con una capa de zinc de pureza no menor a $98 \%$, aplicada por el proceso de inmersión en caliente, según normas internacionalmente reconocidas [BS EN ISO 1461-2009]. Para mantener los derechos de confidencialidad las muestras adoptaron nombres genéricos (tabla 1 ).

Se emplearon muestras de acero galvanizado con y sin recubrimiento, las cuales fueron sometidas a ensayos climáticos acelerados de niebla salina neutra en una cámara climática modelo Q-FOG CCT con capacidad de $1.1 \mathrm{~m}^{3}$, empleándose una solución de cloruro de sodio p.a. $50 \pm 5$ g/l, densidad de la solución de $1.0255-$ $1.0400, \mathrm{pH}$ entre 6.5-7.2, y la temperatura en el interior de la cámara de ensayos es de $35^{\circ} \mathrm{C} \pm 2^{\circ} \mathrm{C}$, con $99-100 \%$ de humedad relativa (UNE EN ISO 9227:2007), y a ensayos de humedad y temperatura con condensación constante en una cámara climática, aplicándose una temperatura de $40 \pm 2^{\circ} \mathrm{C}$ y una humedad relativa entre $99-100 \%$ con condensación constante sobre las probetas de ensayo (UNE EN ISO 6270-2:2006). Las muestras de acero galvanizado fueron sometidas a 500 horas de exposición, por sus condiciones agresivas de explotación (ambiente marino costero y rural expuesto a lluvias 
continuas e intemperismo). Las muestras de acero galvanizado recubiertas se sometieron a 1000 horas de ensayos y, además, al ensayo de envejecimiento acelerado (UNE EN ISO 11507:2007), por la importancia que éste reviste para las pinturas, lacas y barnices en general y la susceptibilidad de estos materiales a la aparición de defectos o modificaciones al ser expuesto a los requerimientos de este ensayo, con la exposición a la radiación ultravioleta fluorescente y al agua. Este ensayo se realizó en una cámara de intemperismo acelerado QUV/SE, para determinar la habilidad del módulo de ser expuesto severamente a las radiaciones UV con lámparas fluorescentes UV de longitud de onda de $280 \mathrm{~nm}$ y un Set Point de $0.7 \mathrm{~W} / \mathrm{m}^{2} / \mathrm{nm}$. Se aplicaron ciclos alternativos hasta alcanzar las 1000 horas de ensayo de 8 horas UV $60^{\circ} \mathrm{C} / 4$ h de condensación a $40^{\circ} \mathrm{C}$. Todas las evaluaciones se efectuaron cada 100 horas y cada resultado es el promedio de tres réplicas de ensayo.

Los requerimientos de composición química reportados para las muestras evaluadas, según Fichas Técnicas de los productores, se corresponden con dos tipos de acero al carbono de baja aleación. Se tomaron las muestras M1 y M2 como representativas de estructuras metálicas (M1) y chapas para conformar (M2), de acero galvanizado; a las cuales se les realizó el análisis de composición química mediante espectrometría de emisión óptica, utilizando para ello un espectrómetro multicanal digital modelo SPECTROLAB.

La figura 1 refleja la muestra M1 en condiciones de explotación y por los elevados requerimientos mecánicos, constructivos y medioambientales a los que está expuesta se le realizó el análisis microestructural, así como a la muestra M2 (ASTM E407:07; ASTM E3:2011).

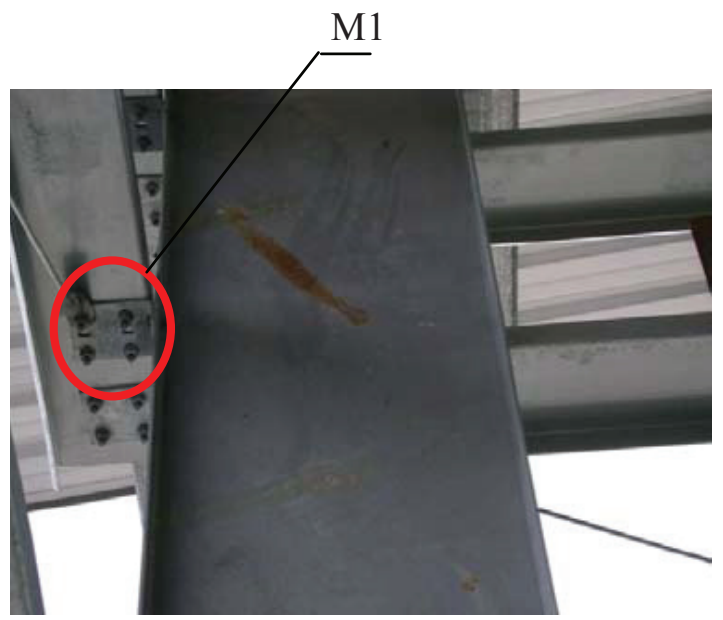

Figura 1. Muestra M1: Estructura metálica de acero galvanizado en explotación
Para ello se empleó un microscopio óptico-metalográfico de lentes planocromáticos y cámara de video a color, determinándose además por esta vía el espesor del recubrimiento. Paralelamente y a modo de comparación, se efectuó la determinación del espesor del recubrimiento por el método magnético (UNE EN ISO 2178: 1996), usualmente más utilizado por ser más económico y, en adelante, el que se empleó para la determinación de espesores del resto de las muestras con superficie plana, utilizando un medidor de espesores con límite superior de medición de 5,000 $\mu \mathrm{m}$ y error de medición de $\pm 1 \%$.

Para completar la caracterización del material base empleado en las muestras representativas, debido a los espesores de las chapas en todos los casos inferiores a 3 $\mathrm{mm}$, se efectuó el ensayo de dureza Vickers (ASTM E92:82, 2003) realizado sobre el sustrato de acero en la superficie de las muestras M1 y M2, saneadas por desbaste, empleando un durómetro Vickers portátil con escala HV/10/15 kgf $/ \mathrm{mm}^{2}$ ). Los resultados se convierten a su equivalente en Brinell con escala HB/2.5/187.5/10 $\mathrm{kgf} / \mathrm{mm}^{2}$ [Kamenichny].

En las muestras de acero galvanizado pintadas se efectuó la determinación de la dureza Buchholz para evaluar la resistencia a la penetración del recubrimiento aplicado (ISO 2815:2003), empleándose para ello un aparato de dureza Buchholz y un microscopio estereoscópico modelo XTL-400 para realizar la lectura. El ensayo de adherencia se realizó según el método de corte por enrejado (UNE EN ISO 2409:2007), utilizando un rayador multicuchillas para 1,2 y $3 \mathrm{~mm}$ y la resistencia a la fisuración durante el plegado se efectuó con un aparato de ensayo de plegado con mandril cilíndrico de 5 mm (NC ISO 1519:2010).

\section{Discusión y análisis de resultados}

Por los valores de composición química obtenidos la muestra M1 se corresponde con un acero aleado al manganeso y la muestra M2 se corresponde con un acero especial para galvanizado $08 \mathrm{Ju}$, reportado para trabajos de conformación en frío y de calidad (tabla 2).

Las muestras fueron observadas con aumento de 100x. Antes del ataque químico, en la muestra M1 se observa una capa de recubrimiento de un espesor promedio de $135 \mu \mathrm{m}$, y después del ataque con nital a $2 \%$, se observó en el núcleo una microestructura de ferrita en forma de granos más perlita formando bandas en el sentido del laminado (figuras 2a y b). En la determinación del espesor del recubrimiento por el método magnético se obtiene un espesor promedio de $145 \mu \mathrm{m}$, clasificando como Z 900 (ASTM A653-2006a). 
Tabla 1. Identificación de las muestras evaluadas

\begin{tabular}{|c|c|}
\hline Muestras & Descripción \\
\hline M1 & Estructura metálica de acero galvanizado \\
\hline M4 & Soportes de tubos de acero galvanizado \\
\hline M5 & Perfil de acero galvanizado I \\
\hline M6 & Perfil de acero galvanizado II \\
\hline M7 & Perfil de acero galvanizado III \\
\hline M8 & Perfil de acero galvanizado IV \\
\hline M9 & Perfil de acero galvanizado $\mathrm{V}$ \\
\hline M11 & Brida I \\
\hline M11a & Brida II \\
\hline M11b & Brida III \\
\hline M11c & Brida IV \\
\hline M12 & Platina Central \\
\hline M12a & Platina Alineador \\
\hline M12b & Platina Extrema \\
\hline M13 & Riostra \\
\hline M16 & Escuadra \\
\hline M17 & Postizo \\
\hline M18 & Suplemento Farola \\
\hline M20 & Brida U \\
\hline M21 & Brida $\mathrm{V}$ \\
\hline M2 & Chapas de acero galvanizado I \\
\hline M22 & Chapas de acero galvanizado II \\
\hline MR23 & Chapas de acero de galvanizado con recubrimiento de pintura electrostática de poliéster I \\
\hline MR24 & Chapas de acero de galvanizado con recubrimiento de pintura electrostática de poliéster II \\
\hline $\mathrm{M} 2 \mathrm{R}$ & $\begin{array}{l}\text { Chapas de acero galvanizado M2 con sistema de pintura con primario epoxi anticorrosiva } \\
\text { y acabado de esmalte de poliuretano) }\end{array}$ \\
\hline MR25 & $\begin{array}{l}\text { Chapas de acero galvanizado M22, con recubrimiento primario y acabado blanco de } \\
\text { esmalte poliuretano }\end{array}$ \\
\hline MR26 & Chapas de acero galvanizado M22, prelacadas y recubiertas con poliéster std \\
\hline MR27 & Perfil de acero galvanizado como refuerzo de perfiles de carpintería de PVC 1 \\
\hline MR28 & Perfil de acero galvanizado como refuerzo de perfiles de carpintería de PVC 2 \\
\hline
\end{tabular}

Tabla 2. Resultado promedio del análisis de composición química efectuado en la muestra M1

\begin{tabular}{cccccccccccc}
\hline Muestra & $\mathrm{C}$ & $\mathrm{Si}$ & $\mathrm{Mn}$ & $\mathrm{P}$ & $\mathrm{S}$ & $\mathrm{Cr}$ & $\mathrm{Ni}$ & $\mathrm{Mo}$ & $\mathrm{Al}$ & Material & Norma \\
\hline M1 & 0.18 & 0.220 & 1.38 & 0.026 & 0.006 & 0.01 & 0.01 & 0.011 & 0.04 & 20G2/ 20Mn2 & GOST/GB \\
M2 & 0.07 & 0.010 & 0.13 & 0.012 & 0.002 & 0.03 & 0.01 & 0.002 & 0.03 & 08Ju/ DX3 & GOST/GB \\
\hline
\end{tabular}
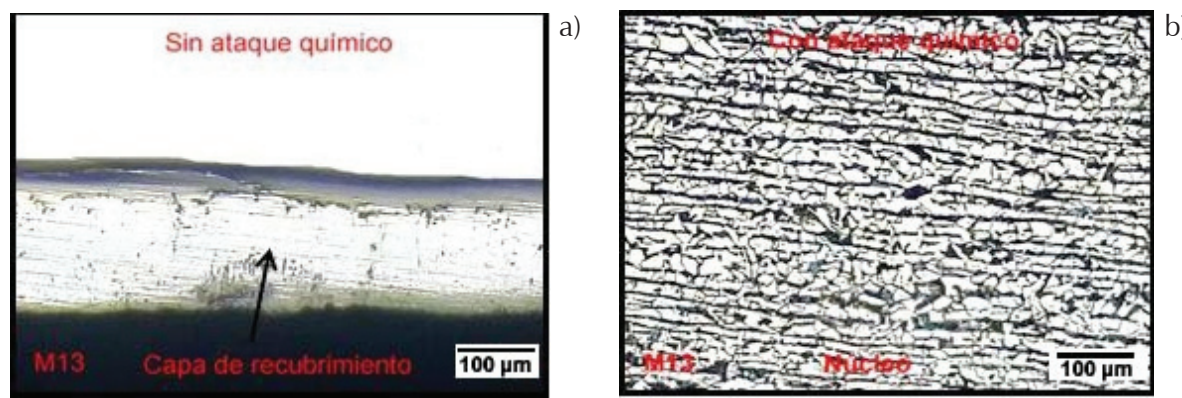

Figura 2. a) Observación microestructural de la muestra M1 del recubrimiento sin ataque y b) el núcleo con ataque con aumentos de $100 x$ 
Antes del ataque químico, en la muestra M2 se observa la capa de recubrimiento de un espesor mucho más reducido, y después del ataque con nital a $2 \%$, se observó en el núcleo una microestructura de ferrita laminar compuesta de granos de ferrita y perlita, con un tamaño de grano ferrito entre 7 y 8 (ASTM E112-1988), (figuras $3 a$ y b). En la determinación del espesor del recubrimiento de la muestra M2 por el método magnético se obtiene un espesor promedio de $22,7 \mu \mathrm{m}$, clasificando como Z 275 (ASTM A653-2006a).

Para la muestra M1, se obtuvo una dureza Vickers promedio mínima de $157 \mathrm{kgf} / \mathrm{mm}^{2}$ y máxima de 170 $\mathrm{kgf} / \mathrm{mm}^{2}$, equivalentes a una dureza Brinell mínima de 158 HB y máxima de 169 HB. El valor obtenido, para la muestra M2, de dureza Vickers promedio mínima es $142 \mathrm{kgf} / \mathrm{mm}^{2}$ y máxima de $169 \mathrm{kgf} / \mathrm{mm}^{2}$, equivalentes a una dureza Brinell mínima de 142 HB y máxima de 169 HB. De acuerdo con los resultados obtenidos, las bases metálicas de las muestras ensayadas se encuentran en estado de procesamiento térmico equivalente al de recocido o normalizado.

Con estos ensayos se caracterizan las muestras M1 y M2 evidenciándose como punto de partida las propiedades del sustrato representativo para la aplicación del galvanizado evaluado.

Las muestras M1 y MR28 se encuentran expuestas en la estación marino-costera de Cojímar, perteneciente al litoral norte de Cuba. La misma ostenta valores de concentración de los principales contaminantes atmosféricos, durante el periodo invierno según registros reportados de: $\mathrm{Cl}^{-} 295.7 \mathrm{mg} / \mathrm{m}^{2} . \mathrm{d} ; \mathrm{SO}_{2} 32.8 \mathrm{mg} / \mathrm{m}^{2}$.d (González y Marrero, 2008).

La influencia del ambiente costero formado por arrecifes, contribuye también a la superior agresividad de esta estación, ya que el efecto de los rompientes en la costa dan lugar a la formación de abundante aerosol marino, lo que unido al marcado efecto transportador de los vientos provenientes del sector norte, cuando tiene lugar la entrada de los frentes fríos y de los vientos alisios del Noreste al Este, son una suma de factores influyentes en la corrosión de los metales y en la degradación de los materiales específicos de este lugar. En el caso particular de Cuba, durante el invierno se experimenta un incremento de la salinidad en la atmósfera, debido a la entrada de los frentes fríos, los cuales vienen acompañados de lluvias y marejadas, ocurriendo en ocasiones lluvias y penetraciones del mar, lo que provoca un incremento en las velocidades de corrosión. y obtiene una categoría de corrosividad atmosférica clasificada como C3, S2P1 según reportes de contaminación referenciados (González y Marrero, 2005). Las muestras fueron colocadas de frente al sur y con un ángulo de inclinación respecto al piso de $30^{\circ}$ (ISO 8565:2011).

La muestra M1 lleva en exposición un periodo de 12 meses y se encuentran aún sin afectaciones. La figura $4 a$, muestra una fotografía de estas muestras en su evaluación tras 12 meses de exposición. Y la muestra MR28, correspondiente al perfil de acero galvanizado sin el recubrimiento protector de PVC, se encuentra expuesta por 17 meses, la figura $4 \mathrm{~b}$ muestra una foto de esta muestra en su evaluación tras 12 meses de exposición, permaneciendo sin afectaciones.

Las muestras correspondientes a chapas de acero galvanizado (M2, M22) y soporte de tubos de aceros galvanizados (M4); no presentaron afectación alguna durante los ensayos climáticos de niebla salina neutra, y de humedad y temperatura con condensación constante, en las condiciones de ensayos experimentadas.

En los resultados del ensayo de determinación del espesor realizados en las diferentes muestras de la gama de productos evaluados (tabla 3), se obtuvieron valores promedios mínimos y máximos de $16.0 \mu \mathrm{m}$ y $145.3 \mu \mathrm{m}$, por lo que las muestras clasifican como G 90 (ASTM A653-2006a) o superior.

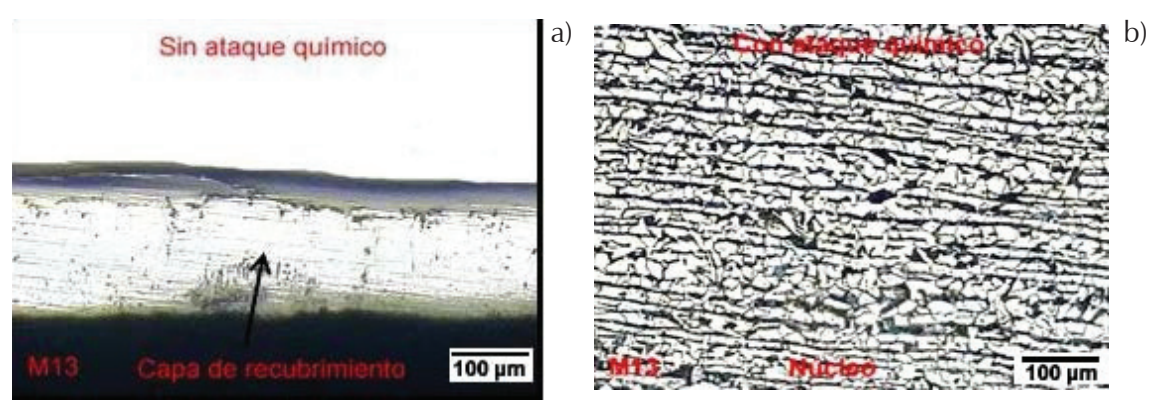

Figura 3. a) Observación microestructural de la muestra M2 del recubrimiento sin ataque y b) recubrimiento con ataque y el núcleo con aumentos de $100 \mathrm{x}$ 

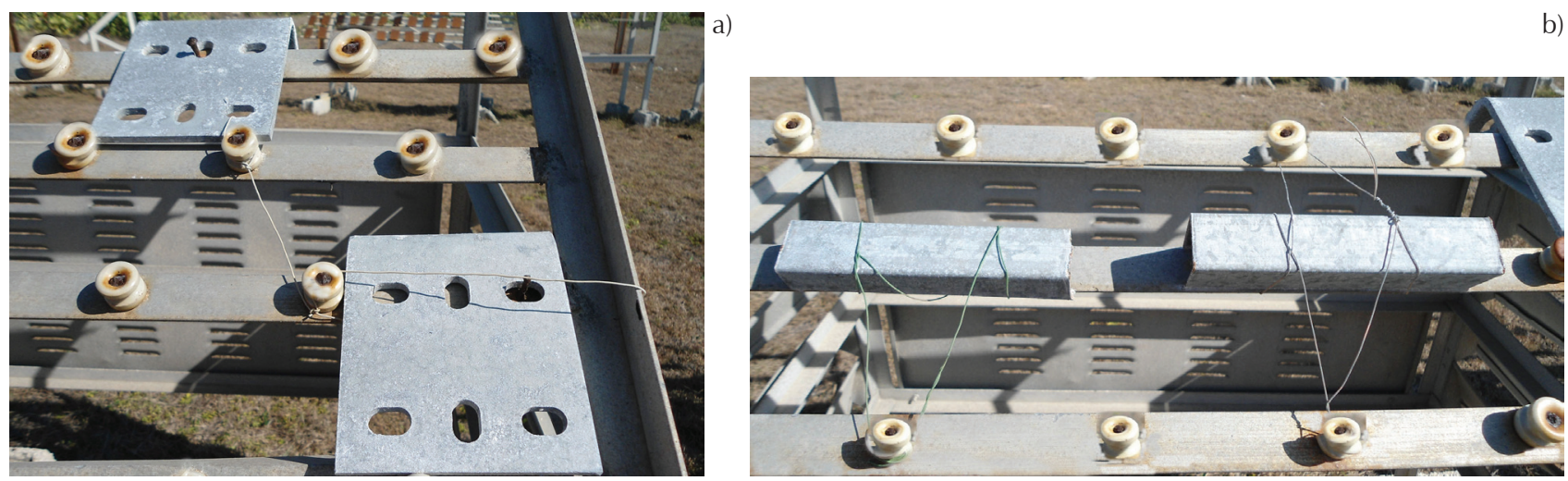

Figura 4. a) Muestra M1, expuesta a 8 meses de ensayo natural en ambiente marino costero, b) muestra MR28, expuesta a 12 meses de ensayo natural en ambiente marino costero

Tabla 3. Determinación del espesor de las muestras galvanizadas

\begin{tabular}{|c|c|c|c|c|c|c|c|c|c|c|c|c|c|c|c|}
\hline Muestra & M1 & M2 & M4 & M7 & M8 & M9 & M11 & M11b & M11c & M12 & M12a & M12b & M17 & M21 & M22 \\
\hline \multirow{3}{*}{$\begin{array}{l}\text { Espesor promedio, } \\
\qquad \mu \mathrm{m}\end{array}$} & 147 & 24.1 & 17.4 & 17.6 & 19.4 & 15.7 & 82.7 & 81.0 & 107 & 96.1 & 121 & 97.4 & 102 & 81.1 & 22.9 \\
\hline & 144 & 22.7 & 20.4 & 22.4 & 19.1 & 15.5 & 89.2 & 55.8 & 108 & 98.8 & 95.2 & 91.8 & 96.5 & 87.1 & 19.9 \\
\hline & 145 & 21.2 & 19.2 & 22.4 & 15.8 & 16.9 & 93.2 & 60.5 & 111 & 119 & 97.9 & 104 & 96.9 & 134 & 22.3 \\
\hline \multirow{3}{*}{ Des. Std. } & 3.2 & 2.8 & 3.7 & 0.1 & 2.2 & 2.1 & 3.7 & 4.9 & 1.9 & 5.8 & 5.2 & 7.5 & 3.3 & 14.0 & 1.1 \\
\hline & 5.9 & 4.3 & 4.1 & 2.3 & 1.8 & 1.0 & 1.6 & 0.1 & 2.6 & 2.0 & 4.1 & 3.9 & 4.8 & 10.0 & 1.3 \\
\hline & 3.9 & 3.6 & 1.5 & 1.5 & 1.9 & 1.3 & 2.4 & 5.3 & 5.2 & 8.6 & 4.4 & 1.4 & 3.8 & 11.9 & 0.1 \\
\hline $\begin{array}{l}\text { Espesor promedio } \\
\text { por muestra, } \mu \mathrm{m}\end{array}$ & 145.3 & 22.7 & 19.0 & 20.8 & 18.1 & 16.0 & 88.4 & 65.8 & 108.7 & 104.6 & 104.7 & 97.7 & 98.5 & 100.7 & 21.7 \\
\hline
\end{tabular}

La muestra correspondiente a la Riostra (M13) presentó signos visibles de afectación a la corrosión a partir de las 300 horas de ensayo. El resto de las muestras de acero galvanizado no presentó afectaciones en el ensayo de niebla salina neutra durante 500 horas. Igualmente, las muestras de acero galvanizado evaluadas no presentaron afectaciones a la inspección visual en el ensayo climático de humedad y temperatura con condensación constante, en las condiciones de ensayos experimentadas.

Por su parte, de las 7 muestras de acero galvanizado recubiertas, sólo las muestras MR27 y MR28 constituidas por perfiles de acero galvanizado como refuerzo de diferentes perfiles de carpintería de PVC, y las muestras MR25 y MR26 correspondientes a un acero galvanizado pintado rebasaron satisfactoriamente el ensayo de niebla salina neutra.

En el caso de las muestras MR23 y MR24, planchas de acero de galvanizado con recubrimiento de pintura electrostática de poliéster, ante el ensayo de niebla salina neutra presentaron un grado de ampollamiento de 2(S2) según norma de referencia (UNE EN ISO 4628-2: 2004), (figuras 5 y 6). Como se aprecia en la figura 6, la muestra MR24 presentó además signos incipientes de corrosión, presumiblemente debido a irregularidades de los aplicadores de pintura y/o a la limpieza previa de la superficie.

Según resultados del ensayo de determinación del espesor realizados en las muestras por el método magnético (tabla 4), se obtienen para MR23 y MR24 espesores promedios del galvanizado de $25.3 \mu \mathrm{m}$ y $27.64 \mu \mathrm{m}$, respectivamente, y un espesor promedio del recubrimiento de pintura de $69.0 \mu \mathrm{m}$ (en espesores de chapa de $0.7 \mathrm{~mm}$ ) y $92.56 \mu \mathrm{m}$ (en espesores de chapa de 1.5 $\mathrm{mm}$ ) para ambas muestras, respectivamente. En particular, según el obtenido para el acero galvanizado, las muestras clasifican como G 90 (ASTM A 653-2006a).

En el ensayo de dureza Buchholz para las muestras recubiertas MR23 y MR24, se obtienen valores promedios de resistencia a la penetración del recubrimiento de 193 y 187, respectivamente. En cambio, en el ensayo de adherencia según el método de corte por enrejado realizado en ambas muestras, se aprecia un área afectada que supera $40 \%$ de la superficie de ensayo, evidenciándose el desprendimiento del recubrimiento en grandes bandas a lo largo de los bordes y/o algunos 


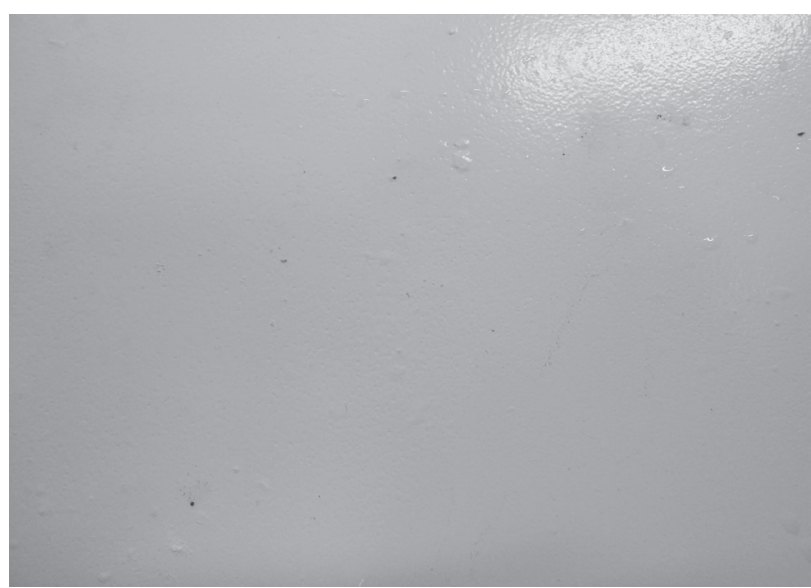

Figura 5. Foto de la muestra MR23, tras 1000 horas de exposición en niebla salina neutra

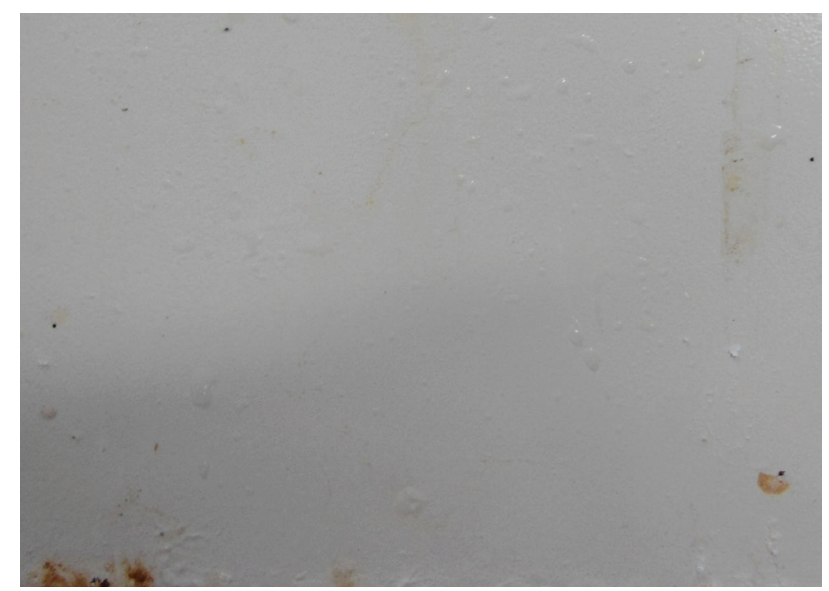

Figura 6. Foto de la muestra MR24, tras 1000 horas de exposición en niebla salina neutra

Tabla 4. Determinación de espesores en las muestras galvanizadas recubiertas

\begin{tabular}{|c|c|c|c|c|}
\hline \multirow[b]{2}{*}{ Muestra } & \multicolumn{2}{|c|}{$\begin{array}{l}\text { Espesor total del recubrimiento en las } \\
\text { chapas de acero galvanizado }\end{array}$} & \multicolumn{2}{|c|}{ Espesor del galvanizado } \\
\hline & Valor Medio, $\mu \mathrm{m}$ & Des. Std. & Valor Medio, $\mu \mathrm{m}$ & Des. Std. \\
\hline \multirow{6}{*}{ MR23 } & 108.0 & 4.8 & 22.3 & 3.1 \\
\hline & 95.5 & 3.9 & 19.7 & 2.1 \\
\hline & 94.6 & 1.9 & 27.4 & 1.3 \\
\hline & 86.5 & 0.1 & 28.1 & 2.3 \\
\hline & 87.1 & 2.2 & 29.2 & 2.0 \\
\hline & 121 & 4.6 & 20.0 & 0.1 \\
\hline \multirow{4}{*}{ MR24 } & 122 & 1.0 & 23.0 & 5.3 \\
\hline & 112 & 4.2 & 39.7 & 1.3 \\
\hline & 125 & 3.4 & 26.5 & 3.3 \\
\hline & 121 & 4.6 & 29.0 & 4.2 \\
\hline \multirow{5}{*}{ M2R } & 98.8 & 1.1 & 24.1 & 2.8 \\
\hline & 99.0 & 5.0 & 22.7 & 4.3 \\
\hline & 89.5 & 3.0 & 21.2 & 3.6 \\
\hline & 81.8 & 2.0 & 25.9 & 3.7 \\
\hline & 87.0 & 3.5 & 21.5 & 2.6 \\
\hline \multirow{5}{*}{ MR25 } & 130 & 3.0 & 22.9 & 1.1 \\
\hline & 138 & 1.1 & 19.9 & 1.3 \\
\hline & 136 & 1.4 & 22.3 & 0.1 \\
\hline & 138 & 2.1 & 17.0 & 1.2 \\
\hline & 132 & 0.1 & 21.2 & 1.9 \\
\hline \multirow{5}{*}{ MR26 } & 42.0 & 0.1 & 22.9 & 1.1 \\
\hline & 44.2 & 1.1 & 19.9 & 1.3 \\
\hline & 42.2 & 0.1 & 22.3 & 0.1 \\
\hline & 38.4 & 0.1 & 17.0 & 1.2 \\
\hline & 38.8 & 1.0 & 21.2 & 1.9 \\
\hline
\end{tabular}


cuadrados desprendidos de forma total o parcial, con clasificación clase 3 según ISO/Clase 2B según ASTM. Obviamente, en el sustrato empleado de acero galvanizado, el recubrimiento no es efectivo al no presentar buena adherencia, ocurriendo un desprendimiento en bandas apreciado en la figura 7 .

En cuanto a la muestra M2R, chapas de acero galvanizado con recubrimiento aplicado, presentaron afectaciones durante el ensayo climático de niebla salina neutra, al apreciarse un ampollamiento visible a la inspección visual a partir de las 300 horas de ensayo. Al concluir las 1000 horas de ensayo el grado de ampollamiento obtenido fue de 2(S2) (figura 8). La muestra M2R no presentó afectaciones en los ensayos de humedad y temperatura con condensación constante e intemperismo acelerado, ante las condiciones de ensayo experimentadas. Los resultados del ensayo de determinación del espesor del recubrimiento (tabla 4), reportaron un valor promedio de $23.08 \mu \mathrm{m}$ para el espesor del galvanizado y de $68.1 \mu \mathrm{m}$ para el espesor del recubrimiento de pintura. El acero galvanizado clasifica como G90 (ASTM A653-2006a). Los resultados de los ensayos de dureza Buchholz y adherencia efectuados no resultaron satisfactorios para un promedio de 3 réplicas, obteniéndose un promedio de dureza Buchholz de 67 y la clasificación de 2, debido a la presencia de ligeros desprendimientos en los bordes según resultado del ensayo de adherencia, con un área afectada no superior a $5 \%$ de la superficie ensayada (figura 9). La determinación del brillo especular evidenció un $47.7 \%$ por lo que la muestra M2R clasifica como categoría II, semibrillante (EN ISO 2813:1999; Qualicoat, 2012).

Las muestras MR25 y MR26, chapas de acero galvanizado y galvanizado prelacado con recubrimiento aplicado, respectivamente; no presentaron afectaciones durante su exposición a los ensayos climáticos acelerados en las condiciones de ensayo experimentadas.

En los ensayos físicos, mecánicos y paramétricos efectuados en las muestras ensayadas, se obtiene para la muestra MR25 un valor promedio de dureza Buchholz de 79, presentando la misma problemas evidentes de fisuración y desprendimiento del recubrimiento en el ensayo de resistencia a la fisuración durante el plegado (figura 10). La muestra MR26 obtuvo valores de dureza Buchholz de 66, ya bastante inferiores al óptimo recomendado por la literatura (Qualicoat, 2012). En la determinación del espesor del recubrimiento (tabla 4), para las muestras MR25 y MR26 se obtienen valores totales promedios de $134.8 \mu \mathrm{m}$ y $41.1 \mu \mathrm{m}$, ambas partiendo de una chapa de acero galvanizado con valor promedio de $20.7 \mu \mathrm{m}$ de espesor del galvanizado clasificando como G90 (ASTM A653- 2006a); garantizándose en MR25 un espesor del recubrimiento aplicado de $114.1 \mu \mathrm{m}$ y para MR26 un prelacado con valores aproximados de $8.3 \mu \mathrm{m}$, para finalmente aplicar recubrimiento de poliéster de espesor promedio de $12.5 \mu \mathrm{m}$. Según la determinación del brillo la muestra MR25 clasifica como categoría II: semibrillante con un 51.5\%, y la muestra MR26 como categoría: mate con un $18.1 \%$. La muestra MR26 no presentó afectaciones en el ensayo de plegado (figura 11).

Por su parte, las muestras MR27 y MR28, correspondientes a perfiles de acero galvanizado como refuerzo de diferentes perfiles de carpintería de PVC, no presentaron afectación alguna durante los ensayos climáticos acelerados de niebla salina neutra y humedad y temperatura con condensación constante. En el ensayo de determinación de espesor por el método magnético

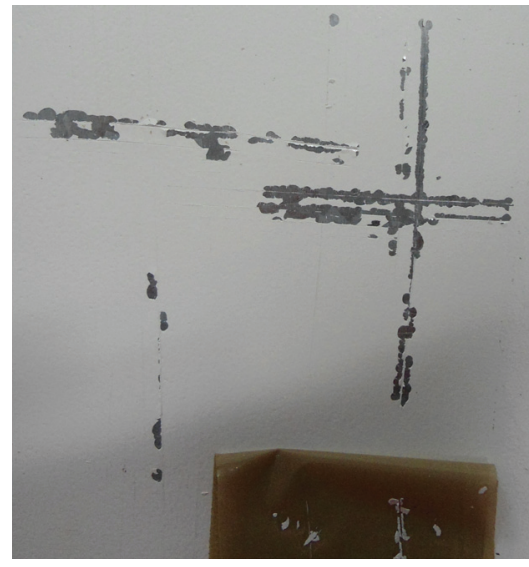

Figura 7. Foto donde se evidencian los resultados del ensayo de adherencia, muestra MR24

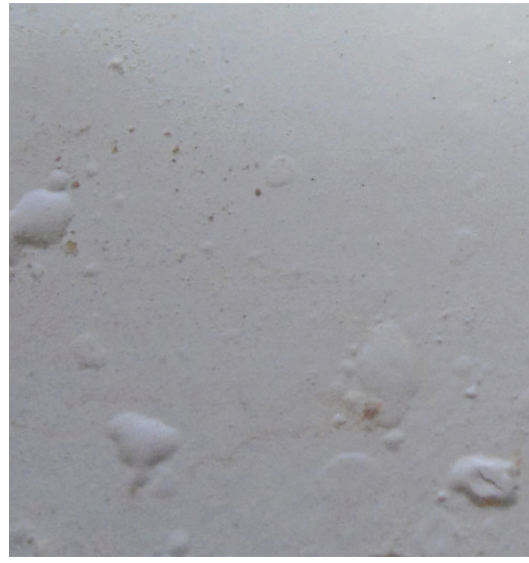

Figura 8. Muestra MR2, expuesta a 1000 horas de niebla salina neutra

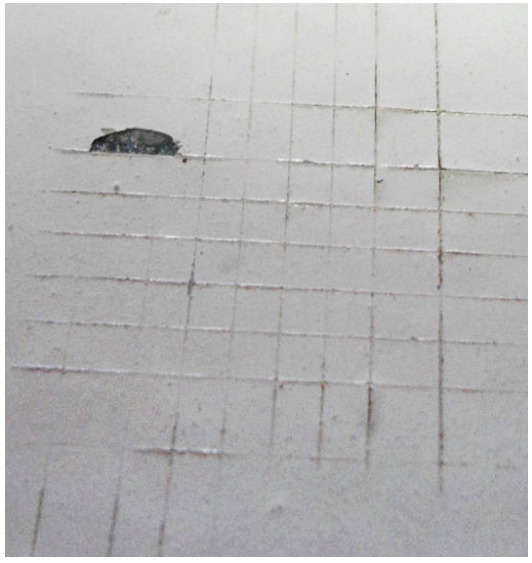

Figura 9. Muestra MR2 posterior al ensayo de adherencia por el método de corte por enrejado 


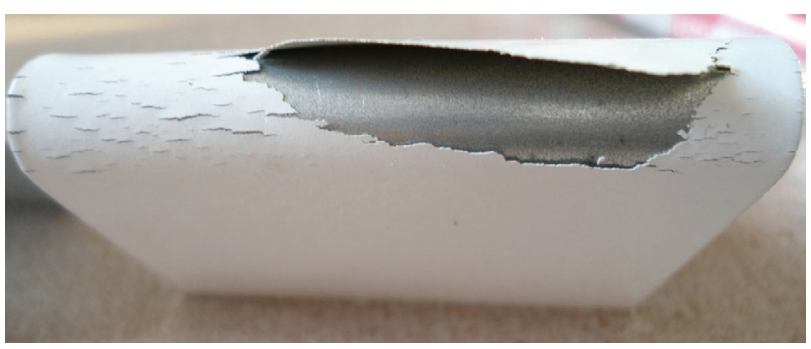

Figura 10. Foto de la muestra MR25, posterior al ensayo de plegado

realizado a estas muestras se obtuvieron valores promedios de 15.92 y $11.62 \mu \mathrm{m}$, respectivamente, siendo clasificados como G 60 (ASTM A653-2006a), de lo cual se puede inferir que esta clasificación es adecuada en ambientes tropicales para aplicaciones en perfiles de acero galvanizado recubiertos con carpintería de PVC, siempre que se garantice que estos perfiles de acero galvanizado estén colocados en el interior de los perfiles plásticos. No obstante, este perfil está expuesto a ensayos naturales en la estación marino costera de Cojímar sin el recubrimiento de PVC para evaluar su comportamiento.

Ninguna de las muestras de acero galvanizado pintadas evaluadas presentó afectaciones durante el ensayo de envejecimiento acelerado, ante la exposición a la radiación ultravioleta fluorescente y al agua.

Estos resultados no son concluyentes, ya que este trabajo se centró en los aceros galvanizados con y sin recubrimiento que están produciéndose o comercializándose actualmente en Cuba. Se debería ampliar el muestreo abarcando valores inferiores de espesores de galvanizado, así como una gama más heterogénea de sistemas de pinturas.

Teniendo en cuenta los resultados obtenidos, podemos inferir que para aplicaciones en climas tropicales húmedos y/o en ambientes marinos costeros expuestas al exterior, es válido el empleo de aceros galvanizados con clasificación G90 (ASTM A653-2006a) o superior, pudiéndose garantizar espesores de sistemas de pinturas adecuados siempre superiores a $100 \mu \mathrm{m}$. Se evidencia igualmente que para aceros galvanizados G90 prelacados con espesores totales de galvanizado y prelacado que garanticen $29 \mu \mathrm{m}$ y un recubrimiento posterior de poliéster aplicado de sólo $12.5 \mu \mathrm{m}$, se logra una excelente resistencia a la corrosión, aún con solo $42 \mu \mathrm{m}$ de espesor total de recubrimiento.

De la misma manera, en los resultados se evidencia que aún teniendo espesores del galvanizado adecuados, cualquiera que sea la variante de sistema de pintura, es importante tener en cuenta por los aplicadores un

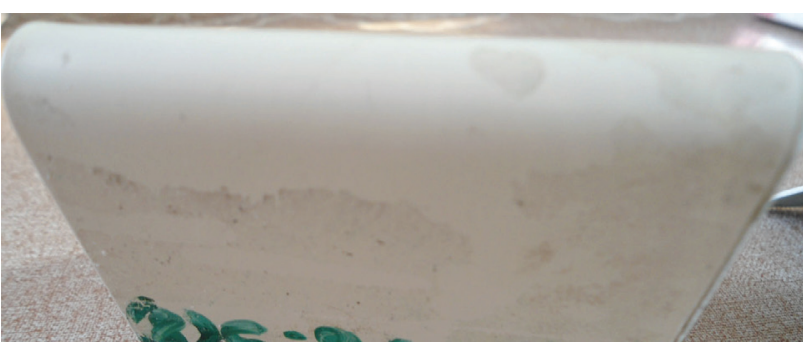

Figura 11. Foto de la muestra MR26, posterior al ensayo de plegado

correcto tratamiento de la superficie y la adecuada aplicación para lograr la debida adherencia del recubrimiento al sustrato y disminuir o minimizar la aparición de defectos indeseables.

\section{Conclusiones}

En el ensayo de niebla salina neutra, con el régimen de ensayos experimentado, $95 \%$ de las muestras de acero galvanizado evaluadas no presentaron afectaciones a la inspección visual.

Según el tamaño de muestra realizado $71 \%$ de los recubrimientos aplicados en aceros galvanizados y en particular $100 \%$ de los aceros galvanizados pintados, no cumplen las exigencias mecánicas y/o corrosivas para las que fueron concebidos, al exponerse a las características específicas del clima tropical húmedo.

De los resultados obtenidos se infiere que para aplicaciones en las condiciones del clima tropical húmedo con atmósfera salina, es válido el empleo de aceros galvanizados con clasificación G90 (ASTM A 653-2006a) o superior, y recubrimientos posteriores y superiores a $100 \mu \mathrm{m}$. Para aceros galvanizados G90 prelacados es factible el empleo de espesores de recubrimientos de poliéster superiores a $12.5 \mu \mathrm{m}$ garantizando un recubrimiento del prelacado de al menos $13 \mu \mathrm{m}$.

Ninguna de las muestras de acero galvanizado con y sin recubrimiento evaluadas, presentaron afectaciones durante los ensayos climáticos de envejecimiento acelerado, y humedad y temperatura con condensación constante.

Se recomienda, para garantizar la eficiencia y rentabilidad de la industria metal-mecánica, lograr elementos de acero galvanizado pintado con las debidas preparaciones de la superficie y aplicación del recubrimiento, prolongándose de esta manera la durabilidad del mismo y garantizándose la tan necesaria relación costo/beneficio, que justificaría la aplicación del recubrimiento. Se debe además; garantizar el mínimo de espesor referido, tanto en la capa del galvanizado como 
en la capa de pintura a aplicar sobre el acero galvanizado dadas las condiciones de explotación del producto, teniendo en cuenta que en condiciones ambientales severas de alta humedad y alta salinidad atmosférica, es muy difícil lograr una protección eficaz del acero galvanizado mediante sistemas de pintura de espesor convencional, aún cuando estén formulados para su aplicación en este tipo de material.

\section{Agradecimientos}

Se agradece la colaboración prestada al Centro de Investigaciones Metalúrgicas de La Habana, por su contribución en la aplicación de algunas técnicas de ensayos, en particular a MSc. José A. Villalonga y los ingenieros Ernesto Espinosa y Medardo Hernández.

\section{Referencias}

Ahmed G., Nishikata A., Tsuru T. Electrochemical Corrosion Monitoring of Galvanized Steel Under Cycle Wet-Dry Condition. Corrosion Science, volumen 42, 2000: 112-120.

ASTM A 653- 06a. Standard Specification for Steel Sheet, ZincCoated (Galvanized) or Zinc-Iron Alloy-Coated (Galvannealed) by the Hot-Dip Process, 2006.

ASTM E 3-11. Standard Guide for Preparation of Metallographic Specimens, 2011.

ASTM E 92-82 (Re: 03). Standard Test Method for Vickers Hardness of Metallic Materials, 2003.

ASTM E 112-88. Standard Test Methods for Determining Average Grain Size, 1988.

ASTM E 407-07. Standard Practice for Microetching Metals and Alloys, 2007.

BS EN ISO 1461-09. Hot Dip Galvanized Coatings on Fabricated Iron and Steel Articles. Specifications and Test Methods, 2009.

Costa J.M. y Vilarrasca M. Effect of Air Pollution on Atmospheric Corrosión of Zinc. Brithish Corrosion Journal, volumen 28 (número 2), 1993: 117.

EN ISO 2813: 99. Measurement of Specular Gloss of Non-Metalic Saint Films at $20^{\circ} \mathrm{C}, 60^{\circ} \mathrm{C}$ and $85^{\circ} \mathrm{C}$, Geneve, Switzerland, 1999.

González-Prada C., Marrero-Águila R. Ensayo climático costero natural para diferentes estructuras de aluminio lacado y anodizado. Revista CNIC. Ciencias Químicas, volumen 39 (número 2), 2008.

González-Prada C., Marrero-Águila R. Factores ambientales y su influencia en la agresividad corrosiva de la estación de ensayos de Cojímar. Comparación con otras estaciones. Revista CENIC. Ciencias Químicas, volumen 36 (número 3), 2005.

Granesse S.L., Rosales B.M. Corrosión y Protección, XVII. Rev. Iberoamericana, volumen 3, (número 197), 1986.

ISO 2815:03. Paints and Barnices-Buchholz Indentation Test, 2003.

Kamenichny I. Heat Treatment a Handbook, Moscú, Mir Publishers, 1980, p. 15.
ISO 8565:2011. Metals and Alloys. Atmospheric Corrosion Testing. General Requirements for Field Test.

Leygraf C. y Graedel T. Atmospheric Corrosion, Electrochemical Society Series, New York, Wiley Interscience, 2000, pp. 329-340.

Morcillo M., Almeida E., Fragata F., Panossian Z. Corrosión y protección de metales en las atmósferas de Iberoamérica. Programa Iberoamericano de Ciencia y Tecnología (CYTED), Madrid, España, Editorial Gráficas Salué, SA, 2002, pp. 203-232.

NC ISO 1519:10 (ISO 1519:02). Pinturas y barnices. Ensayo de plegado, 2010.

Qualicoat, 2012. Directrices concernientes a la marca de calidad para los revestimientos por termolacado del aluminio destinado a la arquitectura, 13a ed., 2012, p.7-17. [en línea] [fecha de consulta: el 2 de febrero de 2013]. Disponible en: www. qualicoat.net.

Townsend H., Gorman C.D., Fischer R.J. Atmospheric Corrosion of Hot Dip Galvanized Bolts for Fastening Weathering Steel Guiderail. Materials Performance, volumen 38 (número 3) 1999: 34-38.

UNE EN ISO 11507:07. Envejecimiento acelerado QUV. Pinturas y Barnices. Exposición de los recubrimientos a envejecimiento artificial. Exposición a la radiación ultravioleta fluorescente y al agua, 2007.

UNE EN ISO 2178:96. Determinación del espesor de recubrimiento sobre sustratos magnéticos, empleando el método magnético, 1996.

UNE EN ISO 2409:07. Ensayo de corte por enrejado. Pinturas y barnices, 2007.

UNE EN ISO 4628-2:04. Pinturas y barnices. Evaluación de la degradación de los recubrimientos. Evaluación del grado de ampollamiento, 2004.

UNE EN ISO 6270-2:06. Determinación de la resistencia a la humedad de probetas en atmósferas con condensación de agua constante, 2006.

UNE EN ISO 9227:07. Secciones 1, 2, 3, 4, 5 y 6 al 12 (apartados 3.1, 3.2, 3.2.1, 3.3, 5.1) y el Anexo A. Ensayos de corrosión en atmósferas artificiales. Ensayos de niebla salina, 2007.

Vázquez A.J. y De Damborenea J.J. Corrosion Resistance of Galvanized Coatings in Rural Environments: Retrospective Evaluation. British Corrosion Journal, volumen 27 (número 4), 1992: 134-139.

Veleva L., Acosta M., Meraz E.. Atmospheric Corrosion of Zinc Induced by Runoff. Corrosion Science Corrosion Science Journal, volumen 51, 2009: 2055-2062 [en línea]. Disponible en: www. elsevier.com/locate/corsci

Vera R.M. Comportamiento frente a la corrosión en ambiente marino de acero galvanizado y acero galvanizado pintado (Duplex). Información Tecnológica. Materiales y Metalurgia, volumen 16, (número 4), 2005: 53-58.

Zhu F., Persson D., Thierry D., Taxen C. Formation of Corrosion Products on Open and Confined Zinc Surfaces Exposed to Periodic Wet/Dry Conditions. Corrosion, volumen 56 (número 12), 2000: 1256-1265. 


\section{Este artículo se cita: \\ Citación estilo Chicago}

Suárez-Corrales, Xenia Isbel, René Valentino Villar-López, Francisco Eduardo Corvo-Pérez, Rigoberto Marrero. Resistencia al clima tropical de aceros galvanizados con y sin recubrimiento. Ingeniería Investigación y Tecnología, XV, 01 (2014): 29-40.

\section{Citación estilo ISO 690}

Suarez-Corrales X.I., Villar-López R.V., Corvo-Pérez F.E., Marrero R. Resistencia al clima tropical de aceros galvanizados con y sin recubrimiento. Ingeniería Investigación y Tecnología, volumen XV (número 1), enero-marzo 2014: 29-40.

\section{Semblanza de los autores}

Xenia Isbel Suárez-Corrales. Master en ciencia y tecnología de materiales con énfasis en la caracterización de los fenómenos de desgaste en materiales metálicos y evaluación de materiales utilizados en la protección de metales contra la corrosión. Investigador agregado, especialista en el Laboratorio de Ensayos de Corrosión, Físico-Químicos y de Materiales del Laboratorio de Ensayos de Tropicalización, Centro de Servicios Tecnológicos, Cuba.

René Valentino Villar-López. Investigador agregado. Especialista principal del Laboratorio de Ensayos de Corrosión, Físico-Químicos y de Materiales del Laboratorio de Ensayos de Tropicalización, Centro de Servicios Tecnológicos, Cuba.

Francisco Eduardo Corvo-Pérez. Doctor en ciencias técnicas en la especialidad de corrosión con énfasis en corrosión atmosférica. Profesor investigador titular en el Centro de Investigación en Corrosión, Universidad Autónoma de Campeche, Campeche, México. Investigador adjunto del Instituto de Ciencia y Tecnología de Materiales, Universidad de La Habana, Cuba.

Rigoberto Marrero. Doctor en ciencias técnicas. Profesor titular y docente de la disciplina ingeniería de los materiales. Jefe del grupo de investigaciones en corrosión de la Facultad de Ingeniería Química del Instituto Superior Politécnico José A. Echeverría. 